\title{
Téoros
}

Revue de recherche en tourisme

\section{La planète du tourisme : quel monde!}

Un essai sur des incertitudes

\section{Marc Laplante}

Volume 6, numéro 3, décembre 1987

Le tourisme, phénomène mondial

URI : https://id.erudit.org/iderudit/1080600ar

DOI : https://doi.org/10.7202/1080600ar

Aller au sommaire du numéro

Éditeur(s)

Université du Québec à Montréal

ISSN

0712-8657 (imprimé)

1923-2705 (numérique)

Découvrir la revue

\section{Citer cet article}

Laplante, M. (1987). La planète du tourisme : quel monde! Un essai sur des incertitudes. Téoros, 6(3), 12-14. https://doi.org/10.7202/1080600ar d'utilisation que vous pouvez consulter en ligne.

https://apropos.erudit.org/fr/usagers/politique-dutilisation/ 


\section{Un essai sur des incertitudes}

\section{La planète du tourisme: quel monde!}

Mon propos est d'appréhender le tourisme comme phénomène planétaire.

Nous sommes habitućs à exprimer en chiffres ces réalités du tourisme. J'ai donc passé de nombreuses heures, longues et ennuyeuses, à éplucher les gros recueils de l'O.M.T., de l'O.C.D.E., de la C.E.E., de I'UNESCO, etc. J'ai aussi parcouru des Almanachs mondiaux $^{(1)}$. Combien voyagent? Qui voyagent? Pour aller où? Pourquoi? Plus les données chiffrées s'accumulent, plus les réponses à ces questions deviennent incertaines. Au Canada seulement, nous connaissons bien la misere des statistiques touristiques pour lesquelles pourtant on entreprend de très vastes enquêtes; alors, quand on veut s'occuper des 203 pays de la planète...

J'ai pensé un moment que la géographie m'offrirait un meilleur panorama: on voyage aujourd'hui sous toutes les longitudes et sous plusieurs latitudes. Mais, à propos du tourisme, la rose des vents est plutốt géopolitique: je sais que le soleil se lève à l'est mais il me faut toujours tenir compte de plusieurs orients: l'extrême, le moyen et le proche; l'occitanie fut le pays de mes ancêtres mais je vois aujourd'hui ce monde de part et d'autre d'un océan, aux prises avec un monde de l'est qui fait bande à part depuis 1917, qui pense et essaie de vivre autrement et qui prétend, comme l'ouest, étendre son règne sur toute la terre; puis il y a l'Afrique que l'est et l'ouest placent au sud pour mieux. se I'arracher; puis enfin, sur la dérive océane, il y a un continent émietté qu'on ne situe plus selon les points cardinaux. Les paysages de la géographie sont des cartes trop complexes pour venir y superposer celles de migrations touristiques.

Alors, j'ai pensé aussi à m'intéresser à la tectonique des plaques! Les spécialistes de cette discipline embrassent toute la planète d'un seul regard pour voir les Amériques s'éloigner du vieux continent et l'Afrique, dériver vers le nord-est... Voila un point de vue global comme celui que j'espérais: mais leurs analyses, finalement, ne s'expriment qu'en frictions et tensions et accordent aux glissements, aux failles et aux fosses une importance extrềme!

"Sociologue, profosceur au Département d'études urbaines et touristiques, UQAM.
Pour voir ce monde de haut et de loin, il me restait un dernier recours: la plongée dans la Poétique de l'espace du philosophe Gaston Bachelard (2). Puisque nous sommes migrants depuis que le monde est monde, puisqu'il a fallu toute la force de tous les Etats pour obtenir une "mise-au-sol " pro" visoire de quelques siècles des forces de travail de l'ère industrielle, nous avons certainement, inscrit dans nos chromosomes, un goût de l'errance et il doit bien sommeiller en chacun de nous un Homme aux semelles de vent ${ }^{(3)}$. Un incessant appel du large jalonne la littérature, savante ou populaire, depuis Marco Polo et Christophe Colomb. Le philosophe qui sait décrypter ces messages comprend que nous soyons déjà sur la lune et que nous partirons bientôt pour une proche planète. Mais qui, aujourd"hui, est prêt à suivre un philosophe! De mes errances personnelles à travers cette variété d'écrits, j'ai retenu surtout que les travaux et les données sur le tourisme international étaient comme emprisonnés sur eux-mêmes, comme fonctionnant dans un système fermé de notions vagues, d'idées reçues, d'énoncés en forme de postulat; j'y ai trouvé aussi un taux assez élevé d"auto-suffisance: le tourisme a été, est et sera ceci et cela, comme si le reste du monde était une quantité négligeable. Sans la moindre ambition de faire avancer la téorologie naissante, je crois devoir communiquer quelques questions pour "remettre à sa place" le tourisme sur la scène du monde. Je dois le faire sous forme d'essai puisque, comme chacun pourra le constater, je navigue dans des eaux troubles...

\section{Tourisme et déplacements}

Trois cents millions de touristes internationaux en 1986; un demi milliard de touristes nationaux: ces chiffres mirobolants sont pourtant modestes puisque les déplacements touristiques ne représentent que $10 \%$ environ du total des déplacements de personnes dans le monde ${ }^{(4)}$.

Le déplacement de personnes: voilà le nouveau concept. Nouveau? Il nous renvoie, à mon avis, à l'ère pré-touristique car il nous dit simplement que les hommes se sont toujours déplacés et le font de plus en plus. Le voyage touristique fut - et reste - un déplacement particulier.

\section{Déplacements ordinaires et luxueux}

Il est significatif, je crois, de rappeler que le concept de déplacement de personnes nous est servi, cette fois-ci, par les portes-paroles des pays du tiers monde qui reçoivent actuellement des touristes. En 1985, se tenait at Paris une conférence internationale de l'O.M.T. (organisation mondiale du tourisme) sur l'adaptation des formations des personnels de tourisme et de voyages. Près de la moitié des deux cents participants venaient des pays en voie de développement. Ayant participé à ces assises, j'ai noté le message très clair des représentants du tiers monde à la grande instance touristique et je le résume ainsi: il faut donner la priorité aux déplacements de personnes - dans les pays en développement - parce que les démarrages, les rattrapages, les modernisations l'exigent. Les gens doivent se déplacer d'abord pour le travail, le commerce, l'éducation, la santé;; ils sont aussi forcé de le faire souvent pour des raisons politiques et militaires, pour des raisons de survie parfois, suite à des cataclysmes naturels; ils le font enfin parce que, là comme ailleurs - et souvent plus qu'ailleurs - les rapports villes-campagnes changent rapidement. Le tiers monde dit finalement qu'il veut ses voies de transports, ses installations d'accueil, ses services aux voyageurs pour ses besoins prioritaires; il conclut laconiquement: si les vacanciers des pays riches veulent toujours venir nous voir, ils seront les bienvenus dans nos installations. En arrière-plan, un autre message: les touristes étrangers auront beaucoup plus de chances de nous connaître s'ils vivent avec nous, s'ils utilisent nos services aux voyageurs! ${ }^{(5)}$ Combien aimeront-ils partager ainsi les conditions de vie de leurs hôtes?

\section{Tourisme et tiers monde:} une expansion bien orchestrée

La réaction récente de certains pays du tiers monde face au tourisme est-elle si surprenante? Qui a voulu cette expansion des activités touristiques sur toute la scène du monde? Contrairement à des idées reçues. cette "aventure" ne commence ni avec les touristes des sociétés industrialisées ni avec les pays en développement; elle fut conçue plutôt comme une stratégie pour assurer l'expansion des sociétés commerciales à la recherche de nouveaux profits. Discours de gauche que tout cela? Cette thèse, quand on la trouve chez des idéologues du tiers monde, emprunte effectivement le ton et l'argumentation typiques du discours anti-impérialisme et anti-capitalisme. Mais elle reçoit également des confirmations de la recherche fondamentale en sociologie du tourisme international. Marie-Françoise Lanfant, qui l'a défendue la première il y a une douzaine 
d'anneses, a dü affronter de forts vents contraires et n'a de cesse de lutter dans son propre pays - la France - pour trouver les moyens de poursuivre ses analyses. Au risque de trop simplifier ses conclusions, je tenterai de résumer ses travaux en quelques énoncés ${ }^{(6):}$

1. Il existe un système d'action touristique intemational qui opere selon une volonte internationale qui trascende les volontés nationales.

2. Pour repérer ce systême, il faut se concentrer sur l'étude des acteurs, des agents et, préalablement à cela, il faut se libérer du carcan des analyses en terthes d'offre et de demande.

3. L'analyse - tres difficile - des agents montre qu'il y a constitution en réseaux à l'échelle mondiale, réseaux interconnectés d'agents, centralisés aux plus hauts niveaux, partageant surtout des méthodes de planification, des modèles d'études et d'evaluation et, de plus en plus, des informations exclusives et stratégiques superbement informatisées a la grandeur du globe et accréditant pour tous les pays, le "know how" de ces centres de décisions ${ }^{17 \%}$.

4. Le système est parvenu à s installer audessus des intérúts nationaux en diffusant une doctrine économique - via les grands organismes officiels - selon laquelle il y a interdépendance d'objectifs entre les pays riches et les pays pauvres.

5. Essentiellement, cette doctrine lie le développement de base des sociétés pauvres au développement luxueux des sociétés riches! Plus la consommation loisir-temps libre s'accroittera dans les pays riches, plus les pauvres en profiteront. Proposer le séjour et la visite touristique dans des pays du tier's monde c'est, d'une part, accélérer la croissance des industries du temps libre dans les sociétés riches et, d'autre part, aider les pays pauvres à trouver des capitaux, à s'équiper, à former des travailleurs, ete.

6. Cette doctrine, qui reçoit ses premières formulations vers 1970 , arrive à point nomme: les promesses de la civilisation du loisir sont difficiles à tenir en pays riches: avec le voyage au bout du monde pour tous, elles retrouveront un nouveau souffle; l'aide aux pays pauvres, faisant appel aux sens de l'équité et aux vieilles idées de la charité, ne trouvait plus beaucoup d'oreilles attentives: la nouvelle doctrine "liberrait" les consciences nationales; les pays riches pouvaient gaspiller à volonté: plus ils le faisaient, plus ils faisaient en même temps du bien aux pauvres!

7. Les sociétés transnationales du tourisme, grands centres de décision de ce système d'action, ont alors mis au point des stratégies d'intégration et d'unifica-

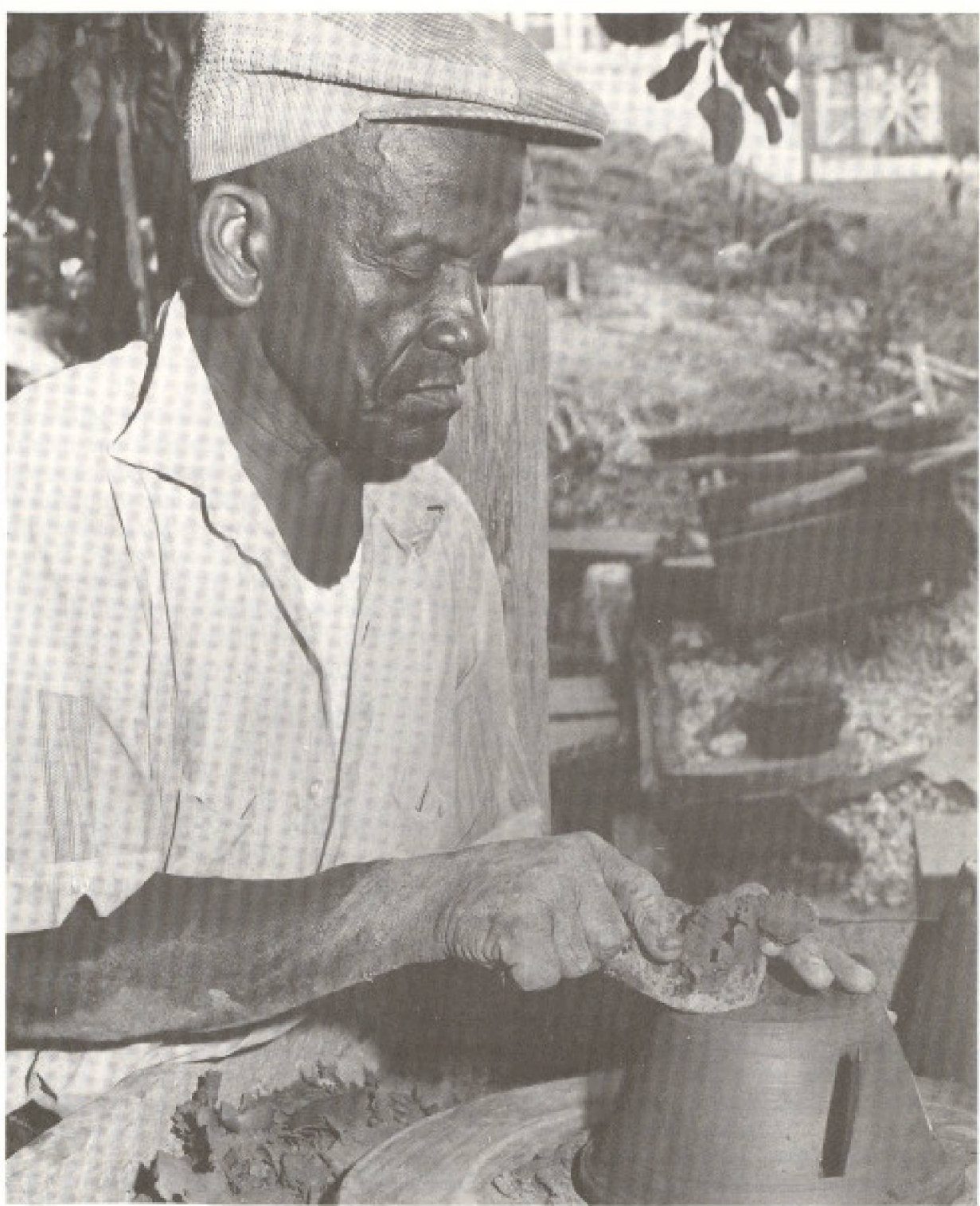

tion de la promotion touristique internationale qui ont connu un succes fabuleux durant une décennic, malgré les crises du petrole. Il a fallu la crise de 1981 pour jeter quelques ombres sur cette opération de grande envergure.

8. Depuis 5 ou 6 ans, le systeme a connu quelques ratés mais les transnationales du tourisme, comme toutes les multinationales, ont su passer ả travers la crise. Je reprendrai ce point, ci-dessous, mais l'analyse de Lanfant soulignait que deux conditions devaient toujours être satisfaites pour que le systeme d'action touristique international marche sur des roulettes: a) il fallait que la consommation-loisir s'aceroisse sans cesse dans les pays riches et b) il fallait que les profits faits dans les pays pauvres y soient ré-investis. Or, d'une part, le niveau de vie des pays industrialisés connaît des aleas et sa croissance assurée devient incertaine, ce qui affecte, en priorité, le budget de vacances et, d'autre part, les retombées du tourisme international dans les pays pauvres sont très loin des espérances des années ' 70 . Les impacts économiques sont souvent théoriques plus que réels et surtout, les conséquences sociales et culturelles du tourisme international dans ces pays sont jugées d'autant plus negatives aujourd'hui qu'elles n'étaient pas dans le "contrat" il y a 15 ans. Même si les experts se perdent encore en conjecture à ce propos, les pays concernés semblent parvenus a leurs conclusions, comme on a pu le voir à la conférence de l'OMT en 1985 a Paris. Le temps des vacances est souvent celui des débordements et des transgressions, comme le temps de la fête auquel il appartient. Ce temps dérange toujours ceux qui ne sont pas de la fete; il devient par contre quasiment scandaleux quand il est vécu sous les yeux de sociétés entières aux prises avec des problemes essentiels de survic et de survivance.

9. Je trahirais trop les dix années d'efforts de recherche de Marie-Françoise Lan- 
fant et de son équipe si je passais sous silence ses travaux plus récents sur les identités. Le tourisme international accentue $1^{+}$épreuve d'identité des sociétés qu'il absorbe dans son système. Ses promoteurs appellent les sociétés locales à affirmer leur identité. Pour les besoins du marketing, ils fabriquent des dépliants et brochures qui présentent les sociétés locales à leurs clientèles, ils fabriquent des produits touristiques qui sont la société elle-même; la production de ces signes identitaires est simple au début: soleil, plage, ete.; plus tard, elle touche des expressions de la culture: les monuments, puis des traces de modes de vie traditionnel, des relations humaines, l'hospitalité, déjà moins palpable et, tôt ou tard, l'intimité: le sexe, le corps, le sacré, les grands rituels. Le Centre (nous des sociétés industrielles) fait pour ses périphéries des productions externes d'identité. Mme Lanfant conclut: "on phantasme l'autre et on échappe d'emblée à l'altérité de l'autre" (cours à l'UQAM).

10. Ces graves questions, posées à l'occasion d'études dans le tiers monde, ne doivent pas faire oublier que le systeme d'action touristique international est mondial. Il agit aussi dans les sociétés développées. Prévoyant par exemple, une réduction possible des voyages à l'étranger, il s'implique dans de nouvelles zones et de nouveaux secteurs dans les sociétés nationales déjà touristiquement développées: les arrières-pays, le tourisme à thèmes, les grands événements, le tourisme industriel, etc. C'est toujours en centrant l'analyse sur les agents et leurs réseaux interconnectés qu'on peut parvenir à suivre à la trace la naissance et l'affirmation de ces nouveaux "courants". Qui a jamais cru qu'ils naissaient spontanément, du pur caprice des innovateurs du tourisme?

\section{Tourisme international: un petit-gros}

Le tourisme international n'est pas si gros, évalué sur l'ensemble des déplacements de personnes dans le monde, mais il n'est pas, pour autant, un phénomène insignifiant car il est porteur de changements. L'internationalisation est un processus qui peut conduire à la mise en place d'un nouveau système social d'échange s'étendant graduellement à toute la terre. Périodiquement - et le président de la France vient encore de le faire durant la semaine noire du 19 octobre 1987 avec le désordre des marchés boursiers - on fait appel à un "nouvel ordre économique mondial", C'est toujours lorsque l'ordre existant a des soubresauts que ce discours se tient. Au niveau des faits l'ordre présent a toujours des ressources pour traverser les crises.

J'avais été fort instruit là-dessus par l'étude de W. Andreff: Les multinationales hors la crise, parue des $1982^{(8)}$. Elle traitait, entre autres sujets, des investissements directs contrôlés par les sociétés multinationales dans le tiers monde: $55 \%$ de tous leurs investissements se faisaient dans 17 pays appelés les "nouveaux pays industriels", par comparaison, ces entreprises ne consacraient que $13 \%$ de leurs investissements aux 77 pays qui occupent le bas de l'échelle des 203 pays représentés à l'ONU, en terme de produit national brut par habitant. (17\% des investissements se trouvaient dans les 17 pays de l'OPEP et $15 \%$, dans une vingtaine de paradis fiscaux: ceci est aussi instructif).

Parallèlement, le livre de P. English: La grande évasion? ${ }^{(9)}$, présentait une liste de 17 pays du tiers monde qui avaient reçu plus d'un million de touristes internationaux en 1982. Ensemble, ces pays avaient accueilli 29 millions de visiteurs cette année-là, soit $53 \%$ du total des arrivées aux frontières dans les pays du tiers monde (selon les statistiques de l'auteur). Mais, faisant le rapprochement avec les données de Andreff, je constatais que 14 des 17 pays du tiers monde recevant le plus de touristes internationaux étaient des "nouveaux pays industriels". J'ose conclure aujourd'hui que, face au tiers monde, les multinationales du tourisme suivent les mêmes stratégies que toutes les multinationales et se sont sorties de la dernière crise de la même façon: contrôle des technologies dites d'avenir, notamment de la transmission des données par satellites, exploitation des divisions du tiers monde qu'elles provoquent en partie et approfondissent à leur profit, durcissement du rapport salarial, etc. Pour celles du tourisme comme pour les autres transnationales, il y a deux tiers mondes, conclut Andreff: l'un en cours d'intégration au capitalisme et l'autre, abandonné à lui-même.

Aucune innocence donc quand la promotion du tourisme international nous parle de la découverte des peuples sympathiques et nous vante les charmes paradisiaques des îles de la ceinture de soleil. J'ai compté, sans trop m'attarder aux situations vécues, une trentaine de micro-sociétés insulaires (Bahamas, Samoa, etc.) et une quinzaine de microsociétés enclavées (Andorre, Lesotho, etc.) recevant au moins cent milles touristes par an; elles sont certes dans la ceinture de soleil (autour du Tropique du Cancer) mais ce qui importe pour moi, c'est que chacune est petite (entre mille habitants: le Vatican, et 1 million, exception faite de la Jamaìque, de Panama, de Hong Kong et de Singapour) et que les recettes touristiques comptent pour $15 \%, 20 \%, 30 \%$ et parfois plus de leur produit national brut respectif (Bahamas: $81 \%$, Antigua: $40 \%$, Iles Vierges, USA: $50 \%$, Maldives: $40 \%$, etc.). Ceci n'est pas tout le tourisme insulaire et enclavé mais il concerne des pays qui ont à décider de leur sort, de façon autonome, en principe.

Dans La Presse récemment, le journaliste Tremblay, arrivant d'Aruba, nouvelle destination plus accessible maintenant "grâce" à American Air Lines, titrait: "faute de pétrole, on espère tout du tourisme" "(10), Ancienne colonie néerlandaise de 65000 habitants, elle obtenait son indépendance au début de 1986, en même temps que Esso pliait bagage après avoir déjà occupé jusqu "à 6500 travailleurs.

Cherchez l'action des agents? La main du grand frère n'est pas toujours invisible: les micro-sociétés de la ceinture de soleil sont souvent des cas faciles à étudier pour qui sait s'interroger. Le tourisme international aux milles visages et aux millions de clients, s'il est très habile, n'est pas toujours le sorcier que l'on pense. $f$

\section{Notes}

(1) L'état du monde, édité chaque année, Paris et Montréal, La décourverte et Boréal; il y a en a d'autres plus spécialisés, comme l'Atlaseco (Paris, Edition SGB]. Pour les organismes internationaux, i'ai consulté surtout leurs rapports annuels des années rócentes.

(2) BACHELARD, Gaston, La poétique de l'espace, Paris, P.U.F., 1957.

(3) LE BRIS, Michel, L'homme aux semelles de vent, Paris, Grasset, 1977. Ce titre, nous dit l'auteur, rappelle comment Verlaine avait appelé Rimbaud.

(4) O.M.T., Conférence internationale sur I'adaptation de la formation des personnels de tourisme et des voyages: Paris, $11-15$ juin 1985 . Voir notam. ment le texte de la Commission A, theme d): "Croissance des mouvements de personnes. des vovages de vacances comme diément moteur contribuant aे la dynamique du développement socio-economique des pays" " (Rapporteur: Vasile Mirces, Roumaniel.

(5) Idem. Voir notamment I'Acte final de la conférence, paragraphe 13: "Le beneflee procure par de tels deplacements t... ceux des vacanciers dans les services pensés pour les besoins nationaux) provient essentiellement de f'enrichissement socio-culturel, psychologique ot economique derivant de la rencontre entre visjteur et visitét.

(6) Je réfère ici particulièrement à Sociologie du tou: risme: positions et perspectives dans le recher che internationale, Paris, C.N.R.S., Centre d'études sociologiques, 1978. Mais j'ai pu consulter la plupart de ces travaux et discuter avec elle durant ses séjours à l'UCAM en 1986 et 1987. Voir: la chronique événement, dans le présent numéro, qui porte sur son centre de recherche: I'URESTI.

(7) Ces réseaux interconnectés sont fort bien décrits dans

François Ascher: Tourisme, sociétés transnationales, et Identités culturelles, Paris, UNESCO. 1904.

(d) Pariš. Le Sycomore.

(9) Ottawa, Institut Nord-Sud, 1986.

(10) TREMBLAY, Michel, La Presse, 24 octobre 1987. page 1.3 . 